\title{
TIP SHAPE DECONVOLUTION OF InSb / GaSb ION BEAM ASSISTED ETCHED SUBSTRATES BY AFM
}

\author{
M. Ospina ${ }^{*}$, S. Vangala ${ }^{* *}$, W. D. Goodhue ${ }^{* *}$, C. Sung ${ }^{*}$ \\ *Department of Chemical Engineering- Center for Advanced Materials; * Department of Physics and \\ Applied Physics- Photonics Center, University of Massachusetts, Lowell, MA 01854
}

\begin{abstract}
Although AFM techniques are very useful in the study of microstructures and surface morphologies the question of whether the experimental AFM image actually reflects the true surface topography, particularly when structures have sharp details is an open question. AFM tips are not infinitely sharp so every image is a convolution of the actual surface topography and the shape of the tip. Before a deconvolution algorithm can be applied, the geometry of the tip must first be determined. There are three ways of determining the AFM tip shape: the use of an electron / field ion emission microscope; deduce the shape of the tip from its image known as a tip characterizer or by the image reconstruction method. In this study, we determined the complex 3D-shape of the tip from non-contact AFM images of etched $\mathrm{InSb}$ and GaSb samples without using a known tip characterizer and then established a simple processing methodology for generating topography images with reduced AFM tip
\end{abstract} contributions by the reconstruction method [1].

Bromine ion beam assisted etching (IBAE) has been successfully used to etch a variety of III-V semiconductors [2]. For this work, non-optimally polished GaSb and InSb surfaces were IBAE etched at room temperature, with a beam voltage $600 \mathrm{~V}$ and cathode current of $3 \mathrm{~mA}$. AFM images were taken at scan sizes of 5, 10 and $25 \mu^{2}$ with a XE-100 AFM, (PSIA Corp.) using the non-contact mode [3]. A scan rate of $0.5-1 \mathrm{~Hz}$, a resolution of $512 \times 512$ pixels, and Si cantilever-tips were used for the measurements.

The AFM images showed sharp cone features formed by the etching technique from the area of interest (Fig 1). A recursive algorithm was employed using commercial AFM deconvolution software to reconstruct the tip shape (Fig 2). We defined the appropriate tip size domain to center the tip apex. Deconvoluted images of the type shown in (Fig 3) indicate a significant narrowing of the cone size. Here the white areas around the cones indicate region of uncertainty in the measurement of the topography due to the finite dimensions of the AFM tip. Because of the nature of the sample (Fig.4) we did not expect significant changes in the surface roughness parameters, RMS and power spectrum density from both experimental and simulated images and none were observed. The certainty of true sample topography calculated was in the range of 74-90\% accuracy. Focusing on the area of the larger cones, we observed that the tip radius is reduced approximately $8 \%$ after deconvolution. Such measurements are extremely important in areas of research such as field emission were the cone geometry is critical. We are currently attempting to measure the actual cone radius with TEM and compare the results to those of deconvoluted AFM images. In conclusion, the current method appeared to be effective and consistent in understanding real tip images from etching experiment for FED applications.

\section{References:}

[1] J.S. Villarrubia, J. Res.Natl. Inst.Stand. Technol.102 (1997)425.

[2] W.D. Goodhue, et al., J. Electron. Mater, 28 (1999) 364.

[3] Joonhyung Kwon, et al., Review of Scientific Instruments, 2002.

[4] This study was supported by the U.S. Defense Advanced Research Projects Agency (DARPA). 

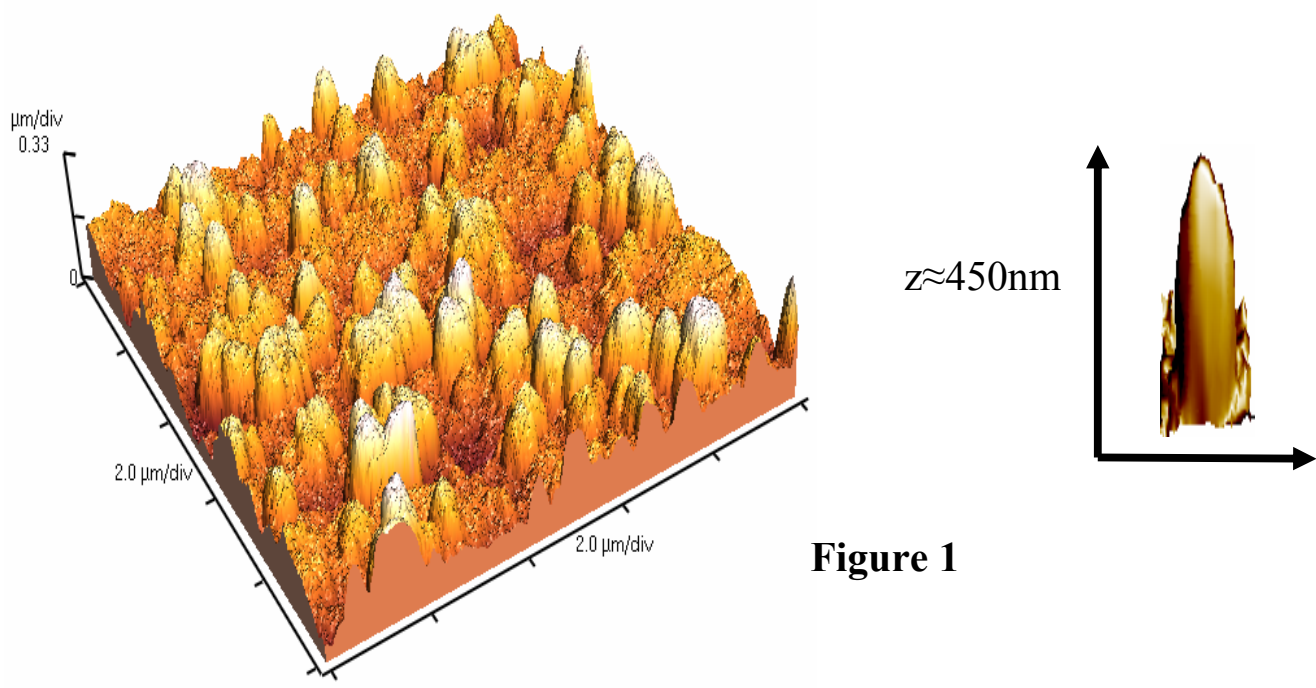

Figure 1

Figure 2

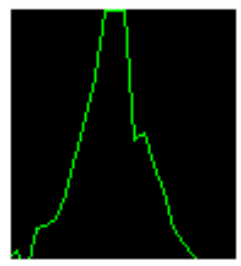

(a)

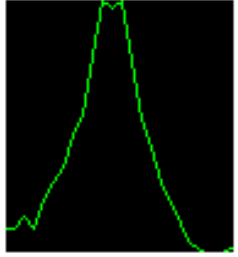

(b)

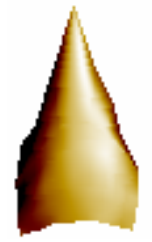

(c)

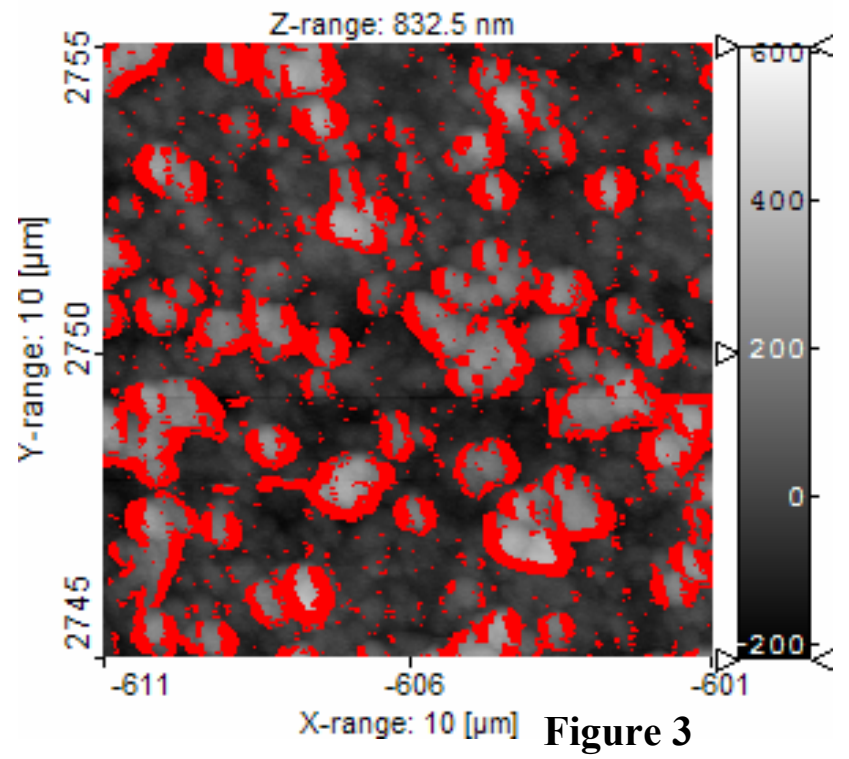

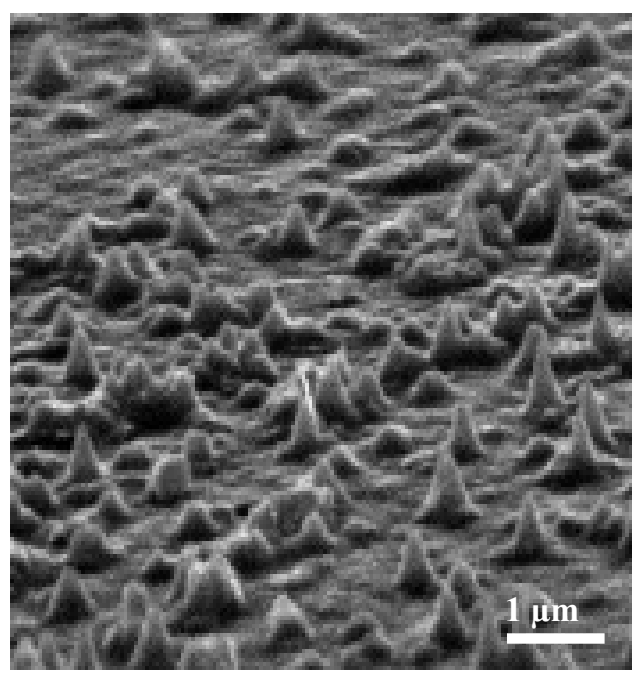

Figure 4

FIG.1 (a) Three-dimensional view of a Non-Contact, 10x10um², AFM topography image (512x512 pixels) of a InSb IBAE processed surface, (b) feature of interest.

FIG.2 (a) X-Z, (b)Y-Z profiles and (c) 3D reconstruction of estimated tip.

FIG.3 Certainty Map of true sample topography (gray area). The white areas around the cones indicate region of uncertainty in the measurement of the topography

FIG.4 SEM image of micro-features of interest taken at $20 \mathrm{KeV}$. 